\title{
Subsite mapping of Hormoconis resinae glucoamylases and their inhibition by gluconolactone
}

\author{
RICHARD FAGERSTRÖM \\ Research Laboratories, Alko Ltd, POB 350, SF-00101 Helsinki, Finland
}

(Received 17 August 1990; revised 14 November 1990; accepted 11 December 1990)

\begin{abstract}
The subsite maps of two purified glucoamylases $(\mathrm{P}$ and $\mathrm{S})$ from the fungus Hormoconis resinae were determined from kinetic data using sets of linear malto-and isomaltooligosaccharides as substrates. Glucoamylase $P$, which has an unusually high debranching (1,6-glucosidic) activity, showed a subsite map different from all known subsite maps of glucoamylases. The free energy of binding of maltooligosaccharides was negative at subsite 1 , whereas all the others show a positive or zero energy at subsite 1. Inhibition of both glucoamylases $P$ and $S$ acting on either malto- or isomaltohexaose by gluconolactone [D-glucono-(1,5)-lactone] was investigated. Gluconolactone decreased the values of the maximum velocity, $V$, suggesting it can bind to subsite 1 . The size of inhibition constants, identified as dissociation constants of gluconolactone from free enzyme, depended on whether the substrate was maltohexaose or isomaltohexaose. This suggests that gluconolactone has at least two binding sites, and that there are different subsites 1 (or 2) for 1,4- and 1,6-linked substrates. From previously reported results with other glucoamylases, an 'induced fit' model was constructed for glucoamylases hydrolysing oligosaccharides.
\end{abstract}

\section{Introduction}

Glucoamylases (1,4- $\alpha$-D-glucan glucohydrolases, EC 3.2.1.3) are enzymes that degrade oligosaccharides such as amylose and amylopectin, releasing D-glucose from the nonreducing-end of the oligosaccharide. Usually the activity towards the branching points (1,6and 1,3-glucosidic bonds) of natural oligosaccharides is low. However, some glucoamylases do contain high debranching activity, and these enzymes are of commercial interest for the food and fermentation industry in that they are capable of converting their oligosaccharide substrates almost completely to glucose.

Hiromi (Hiromi, 1970; Hiromi et al., 1973a) has developed a subsite theory for analysing the substrate affinities of glucoamylases. The active site of glucoamylase is proposed to consist of several subsites, which are numbered starting from the binding site of the glucose at the nonreducing-end. The basic assumptions made are as follows. 1. The substrates bind in rapid equilibria. 2. The active site consists of $n$ subsites, each having an affinity $A_{n}$ for a residue of the substrate. 3. A substrate can be bound to the enzyme in either productive or nonproductive modes. 4 . The intrinsic rate $\left(k_{\text {int }}\right)$ of hydrolysis is independent of substrate length. 5. The binding affinity of a oligomeric substrate is equal to the sum of the individual subsite affinities.
The subsite structure of different glucoamylases seems to have a common arrangement, where subsite 2 has the highest affinity for the oligomeric substrates and glucose (Ohnishi et al., 1975; Hiromi et al., 1973a), followed by a decreasing affinity upon going towards subsites 3 to 7 . Subsite 1 seems to show a negative or zero affinity (Tanaka et al., 1983a).

The cleavage of different $\alpha$-D-glycosidic bonds is considered to occur at the same active centre of glucoamylases (Hiromi et al., 1966a,b,c), between subsites 1 and 2 (Hiromi et al., 1973a).

Gluconolactone [D-glucono-(1,5)-lactone], having a distorted half-chair conformation, has been considered to be a transition state analogue in the hydrolysis reaction (Ohnishi et al., 1975; Hiromi et al., 1983). Studies of glucoamylases from Rhizopus niveus and Rhizopus delemar have suggested that this inhibitor binds to subsite 1 of these enzymes (Ohnishi et al., 1975; Hiromi et al., 1982, 1983; Tanaka et al., 1982).

The amino acid sequences of six different microbial glucoamylases have been determined. The complete sequences of Aspergillus niger (Svensson et al., 1983) and Aspergillus awamori (Nunberg et al., 1984) glucoamylases are identical. The glucoamylases from Rhizopus oryzae (Ashikari et al., 1986), Saccharomyces diastaticus (Yamashita et al., 1985), Saccharomyces cerevisiae (Yamashita et al., 1987) and Saccharomycopsis fibuligera (Itoh et al., 
1987) share four highly conserved regions with the Aspergillus enzyme (Tanaka et al., 1986; Itoh et al.,1987). These regions, numbered according to the sequence of $A$. awamori glucoamylase, include Asp-176, Glu-179, Glu180 and Trp-120. Sierks et al. (1990) have, on the basis of site-directed mutation experiments, suggested Glu-179 as the general acid catalyst and Asp-176 as the general base catalyst in the hydrolysis reaction of oligosaccharides. Glu-180 plays, according to their interpretation, a role in raising the $\mathrm{p} K_{\mathrm{i}}$ of Glu-179. Fluorescence quenching of one or more tryptophanyl residues upon binding of substrate to glucoamylases has been demonstrated (Hiromi et al., 1982; Clarke \& Svensson, 1984). Trp-120 is proposed to be needed for the stabilization of the transition-state complex in subsites 1 and 2 (Sierks et al., 1989).

The fungus Hormoconis resinae (ATCC 20495) produces two extracellular glucoamylases ( $P$ and $S)$, one of them $(\mathrm{P})$ having an exceptionally high activity towards 1,6-glycosidic bonds in addition to the 1,4-glycosidic activity (McCleary \& Anderson, 1980). These glucoamylases seem to be highly homologous (Fagerström et al., 1990), and also to show high homology to other glucoamylases (to be published elsewhere), especially in the four highly conserved regions.

\section{Methods}

Materials. Linear oligosaccharides ranging from maltose (4- $O-\alpha-\mathrm{D}-$ glucopyranosyl-maltose) to maltoheptaose (4-O- $\alpha$-D-glucopyranosylmaltoheptaose) (Sigma) and isomaltose (6-O- $\alpha$-D-glucopyranosylisomaltose) to isomaltoheptaose (6- $O$ - $\alpha$-D-glucopyranosyl-isomaltoheptaose) (Biocarb, Uppsala, Sweden) were used as substrates. To determine released glucose, a kit based on glucose dehydrogenase (Merck) was used. In the case of gluconolactone inhibition, released glucose was measured with a kit based on hexokinase (Boehringer).

Enzyme preparations. Glucoamylase $\mathrm{P}$ and $\mathrm{S}$ were purified from Hormoconis resinae (ATCC 20495) culture media as described in Fagerström et al. (1990). The preparations were homogeneous on SDSPAGE. The apparent molecular masses estimated from SDS-PAGE were $71 \mathrm{kDa}$ (glucoamylase $\mathrm{P}$ ) and $78 \mathrm{kDa}$ (glucoamylase $\mathrm{S}$ ).

The concentration of purified protein was determined spectrophotometrically at $205 \mathrm{~nm}$ using the method described by Scopes (1974); $A_{1 \mathrm{~cm}}^{1 \%}$ values of 307 for glucoamylase $P$ and 314 for glucoamylase $S$ were used.

Determination of subsite energies. The kinetic parameters, $K_{\mathrm{m}}$ and $V$ were determined by incubating enzyme with the respective linear oligosaccharides. Reactions were performed at $25^{\circ} \mathrm{C}$. The incubation buffer was $50 \mathrm{~mm}$-sodium acetate, $\mathrm{pH} 4 \cdot 30$, containing $150 \mu \mathrm{g}$ ovalbumin (Sigma grade $\mathrm{V}$ ) $\mathrm{ml}^{-1}$. In order to minimize the formation of oligosaccharides from D-glucose by the condensation reaction of glucoamylase (Nikolov et al., 1989), short incubation times $(0.5$ to $15 \mathrm{~min}$ ) were used. In the case of glucoamylase $\mathrm{S}$ with isomaltooligosaccharides, however, incubation times of up to $40 \mathrm{~min}$ had to be used. Samples were withdrawn at 0.5 to $10 \mathrm{~min}$ intervals, heated at $100^{\circ} \mathrm{C}$ for $3 \mathrm{~min}$ and then transferred to an ice-water bath.
The amount of glucose released was measured with the glucose dehydrogenase kit. All incubations with the different substrate concentrations were run as duplicates, and in the case of glucoamylase $\mathbf{P}$ with malto- and isomaltooligosaccharides, two independent experiments were performed.

To minimize the effect of substrate. hydrolysis producing a new shorter substrate for the next hydrolysis round, only points at less than $14 \%$ complete theoretical hydrolysis were accepted. Substrate concentrations ranged from about half $K_{\mathrm{m}}$ to at least $10 \times K_{\mathrm{m}}$, except for glucoamylase $\mathrm{S}$ with isomaltooligosaccharides, where substrate concentrations of up to $5 \times K_{\mathrm{m}}$ were used. The kinetic parameters were calculated from the initial velocity data according to Wilkinson (1961) (steady-state kinetics) using nonlinear regression analysis (GaussNewton, no weighting, sAs software). Values for molecular activity, $k_{0}$ [from the molar concentration of enzyme $\left(e_{0}\right)$ and maximum velocity $(V), k_{0}=V / e_{0}$ and $k_{0} / K_{\mathrm{m}}$ were calculated and used to determinate the free energies of binding for each subsite 3 to 7 by using equation (1). For estimating the intrinsic rate, $k_{\text {int }}$, and the free energy of substrate binding at subsite $1\left(A_{1}\right)$, equation (2) was used [by plotting $e^{\left(A_{n}+1: R T\right)}$ against $\left.\left(1 / k_{0}\right)_{n}\right]$.

The free energy of substrate binding at subsite 2 was calculated from $K_{\mathrm{m}}$ by using equation (3) and also from $k_{\mathrm{int}}$ and $k_{0} / K_{\mathrm{m}}$ using equation (4). For the derivation of these equations, the reader is referred to Hiromi et al. (1973a).

$$
\begin{aligned}
& A_{n+1}=\boldsymbol{R} T\left[\ln \left(k_{0} / K_{\mathrm{m}}\right)_{n+1}-\ln \left(k_{0} / K_{\mathrm{m}}\right)_{n}\right] \\
& e^{\left(A_{n+1} / R T\right)}=\left\{\left[k_{\mathrm{int}} /\left(k_{0}\right)_{n}\right]-1\right\} e^{\left(A_{A} / R T\right)} \\
& A_{2}=-\boldsymbol{R} T \ln \left\{0 \cdot 018\left(K_{\mathrm{m}}\right)_{n}\left[e^{\left(A_{1}+A_{3}+\ldots A_{n}\right) / R T}+e^{\left(A_{4}+A_{4}+\ldots A_{n+1}\right) / \boldsymbol{R} T}\right]\right\} \\
& A_{2}=\boldsymbol{R} T \ln \left[\left(k_{0} / K_{\mathrm{m}}\right)_{n} /\left(0.018 \times k_{\mathrm{int}}\right)\right]-\left(A_{1}+A_{3}+\ldots+A_{n}\right)
\end{aligned}
$$

Inhibition by D-glucono-(1,5)-lactone. Maltohexaose and isomaltohexaose were incubated with enzyme at $25^{\circ} \mathrm{C}$ with or without the inhibitor D-glucono-(1,5)-lactone (gluconolactone) in $50 \mathrm{~mm}$-sodium acetate, $\mathrm{pH} 4 \cdot 3$, containing ovalbumin $\left(150 \mu \mathrm{g} \mathrm{ml} \mathrm{m}^{-1}\right)$. Samples of $60 \mu \mathrm{l}$ were withdrawn at 3 to $20 \mathrm{~min}$ intervals, heated at $100^{\circ} \mathrm{C}$ for $3 \mathrm{~min}$ and cooled in an ice-water bath. The samples were assayed for released glucose by the hexokinase method. Gluconolactone solutions were prepared just before use (less than $5 \mathrm{~min}$ ) to minimize the possible conversion to D-glucono-(1,4)-lactone or hydrolysis to gluconic acid (Shimihara \& Takahashi, 1970).

\section{Results}

The $K_{\mathrm{m}}, k_{0}, k_{0} / K_{\mathrm{m}}$ and $k_{\text {int }}$ values of the glucoamylases with linear maltooligosaccharides (G2 to G7) and linear isomaltooligosaccharides (IG2 to IG7) as substrates are listed in Table 1. The calculated free energies of substrate binding $\left(A_{n}\right)$, together with the estimates of the 'worst case' combinations of errors in the six estimates of $K_{\mathrm{m}}$ $( \pm 20 \%)$ and six estimates of $V( \pm 7 \%)$, are listed in Table 2 (assuming $A_{8}$ to be zero). Values for the second subsite calculated by the two different methods were essentially the same, and their average was used in each case for $\mathrm{A}_{2}$.

The recalculated kinetic parameters from the free energies of substrate binding were in good agreement with the observed ones (not shown; the deviations were between $0 \%$ and $20 \%$ ). Thus the theory yields an internally consistent set of subsite affinities for $H$. resinae glucoamylases. 
Table 1. Kinetic data for glucoamylases $P$ and $S$

The kinetic values $K_{\mathrm{m}}$ and $k_{0}\left(=V / e_{0}\right)$ for both glucoamylases acting on either malto- or isomaltooligosaccharides of different length $(n)$, were obtained from initial velocity data using nonlinear regression analysis as described in the text. In addition, the calculated intrinsic rates of hydrolysis $\left(k_{\text {in }}\right)$ are shown.

\begin{tabular}{|c|c|c|c|c|c|c|}
\hline \multirow[b]{2}{*}{$\begin{array}{l}\text { Value of } \\
n\end{array}$} & \multicolumn{3}{|c|}{$\begin{array}{l}\text { Maltooligosaccharides } \\
\text { (G) }\end{array}$} & \multicolumn{3}{|c|}{$\begin{array}{l}\text { Isomaltooligosaccharides } \\
\text { (IG) }\end{array}$} \\
\hline & $\underset{(\mathrm{mM})}{K_{\mathrm{m}}}$ & $\begin{array}{c}k_{0} \\
\left(s^{-1}\right)\end{array}$ & $\begin{array}{c}k_{0} / K_{\mathrm{m}} \\
\left(\mathrm{s}^{-1} \mathrm{mM}^{-1}\right)\end{array}$ & $\begin{array}{c}K_{\mathrm{m}} \\
(\mathrm{mM})\end{array}$ & $\begin{array}{c}k_{0} \\
\left(\mathrm{~s}^{-1}\right)\end{array}$ & $\begin{array}{c}k_{0} / K_{\mathrm{m}} \\
\left(\mathrm{s}^{-1} \mathrm{mM}^{-1}\right)\end{array}$ \\
\hline \multicolumn{7}{|c|}{ Glucoamylase P } \\
\hline 2 & $1 \cdot 108$ & 3.92 & $3 \cdot 53$ & $14 \cdot 55$ & 1.92 & $0 \cdot 13$ \\
\hline 3 & $0 \cdot 174$ & $11 \cdot 60$ & $66 \cdot 58$ & $4 \cdot 523$ & $2 \cdot 76$ & 0.61 \\
\hline 4 & $0 \cdot 145$ & $19 \cdot 43$ & 133.67 & $3 \cdot 158$ & $5 \cdot 35$ & 1.70 \\
\hline 5 & $0 \cdot 133$ & $13 \cdot 26$ & $99 \cdot 45$ & $2 \cdot 840$ & $6 \cdot 53$ & $2 \cdot 29$ \\
\hline 6 & $0 \cdot 111$ & 17.07 & $153 \cdot 27$ & $2 \cdot 585$ & $7 \cdot 37$ & $2 \cdot 85$ \\
\hline 7 & $0 \cdot 086$ & $19 \cdot 08$ & $222 \cdot 67$ & $2 \cdot 127$ & $5 \cdot 52$ & 3.07 \\
\hline & \multicolumn{3}{|c|}{$k_{\mathrm{int}}=19.4$} & \multicolumn{2}{|c|}{$k_{\mathrm{int}}=30.8$} & \\
\hline \multicolumn{7}{|c|}{ Glucoamylase $S$} \\
\hline 2 & $1 \cdot 420$ & $5 \cdot 03$ & $3 \cdot 54$ & $36 \cdot 50$ & $0 \cdot 160$ & 0.0044 \\
\hline 3 & 0.609 & $29 \cdot 41$ & $48 \cdot 29$ & $22 \cdot 24$ & $0 \cdot 523$ & 0.0234 \\
\hline 4 & 0.395 & $40 \cdot 27$ & 101.94 & 24.08 & 0.825 & 0.0342 \\
\hline 5 & $0 \cdot 235$ & $42 \cdot 66$ & $181 \cdot 51$ & $22 \cdot 65$ & 0.754 & 0.0333 \\
\hline 6 & $0 \cdot 177$ & $48 \cdot 33$ & 273.06 & $24 \cdot 34$ & 0.814 & 0.0334 \\
\hline 7 & $0 \cdot 206$ & $49 \cdot 83$ & $241 \cdot 87$ & $24 \cdot 15$ & 0.753 & 0.0312 \\
\hline & \multicolumn{2}{|c|}{$k_{\mathrm{int}}=261 \cdot 6$} & \multicolumn{4}{|c|}{$k_{\text {int }}=5 \cdot 4$} \\
\hline
\end{tabular}

For both enzymes, the effect of increasing chain length in either oligosaccharide series was to increase $k_{0}$ (by 3to 10-fold) and decrease $K_{\mathrm{m}}$ (by 7- to 11-fold, except that the $K_{\mathrm{m}}$ of glucoamylase $\mathrm{S}$ for isomaltooligosaccharides did not decrease further after isomaltotriose). These trends were usually monotonic, but, for example, glucoamylase $\mathrm{P}$ showed a peak $k_{0}$ at both maltotetraose and maltoheptaose. However, in general the larger substrates were both more tightly bound and more rapidly hydrolysed.

The differences between the $k_{0}$ values for the oligosaccharides of different length, compared to the $k_{\text {int }}$ value of the respective enzyme, can be explained by the distribution of productive and nonproductive binding (Suetsugu et al., 1973). In the cases of glucoamylase P and $\mathrm{S}$ with isomaltooligosaccharides and glucoamylase $\mathrm{S}$ with maltooligosaccharides, the $k_{0}$ values are much smaller than the corresponding $k_{\text {int }}$. The substrates seem to be bound preferentially in nonproductive modes. Only glucoamylase $\mathrm{P}$ with maltooligosaccharides as substrate showed a $k_{0}$ (at G4 and G7) of the same size as $k_{\text {int }}$, indicating that in these cases productive complexes overwhelm nonproductive complexes.

Because for all substrates $A_{2}$ (the free energy of binding at subsite 2) is much more negative than $A_{3}$, even than the sum of $A_{3}+A_{4} \ldots A_{7}$, the most important nonproductive complex will occupy subsite 2 . The
Table 2. Free energy of binding of substrate to glucoamylases $P$ and $S$

The minimum and maximum estimates given show the effect of 'worst case' combinations of errors in the six estimates of $K_{\mathrm{m}}$ $( \pm 20 \%)$ and the six estimates of $V( \pm 7 \%)$ and generate each series of subsite binding energies.

\begin{tabular}{|c|c|c|c|c|c|c|}
\hline \multirow{3}{*}{$\begin{array}{l}\text { Value of } \\
A_{n}\end{array}$} & \multicolumn{6}{|c|}{ Substrate binding energy $\left(\mathrm{kJ} \mathrm{mol}^{-1}\right)$} \\
\hline & \multicolumn{3}{|c|}{ Glucoamylase P } & \multicolumn{3}{|c|}{ Glucoamylase S } \\
\hline & Mean & Max. & Min. & Mean & Max. & Min. \\
\hline \multicolumn{7}{|c|}{ Maltooligosaccharides } \\
\hline 1 & -3.83 & $-5 \cdot 63$ & -0.98 & $+3 \cdot 27$ & $-1 \cdot 48$ & $+25 \cdot 08$ \\
\hline 2 & $-19 \cdot 04$ & $-22 \cdot 43$ & $-16 \cdot 88$ & $-19 \cdot 70$ & $-22 \cdot 08$ & $-17 \cdot 22$ \\
\hline 3 & $-7 \cdot 27$ & $-8 \cdot 62$ & $-5 \cdot 92$ & -6.47 & $-7 \cdot 82$ & $-5 \cdot 12$ \\
\hline 4 & $-1 \cdot 73$ & -3.08 & -0.37 & -1.85 & $-3 \cdot 20$ & $-0 \cdot 50$ \\
\hline 5 & +0.73 & -0.62 & $+2 \cdot 08$ & $-1 \cdot 43$ & $-2 \cdot 78$ & $-0 \cdot 08$ \\
\hline 6 & -1.07 & $-2 \cdot 42$ & $+0 \cdot 28$ & $-1 \cdot 01$ & $-2 \cdot 36$ & $+0 \cdot 34$ \\
\hline 7 & -0.92 & $-2 \cdot 28$ & +0.44 & $+0 \cdot 30$ & $-1 \cdot 05$ & +1.65 \\
\hline \multicolumn{7}{|c|}{ Isomaltooligosaccharides } \\
\hline 1 & +3.00 & -1.72 & $+22 \cdot 57$ & +4.49 & -0.81 & $+23 \cdot 6$ \\
\hline 2 & -16.57 & $-18 \cdot 89$ & $-14 \cdot 02$ & -13.95 & $-15 \cdot 89$ & $-11 \cdot 07$ \\
\hline 3 & -3.80 & $-5 \cdot 15$ & $-2 \cdot 45$ & $-4 \cdot 16$ & $-5 \cdot 52$ & $-2 \cdot 81$ \\
\hline 4 & $-2 \cdot 53$ & -3.88 & $-1 \cdot 18$ & -0.93 & $-2 \cdot 28$ & -0.42 \\
\hline 5 & -0.75 & $-2 \cdot 10$ & $+0 \cdot 60$ & +0.07 & $-1 \cdot 28$ & $+1 \cdot 42$ \\
\hline 6 & -0.54 & $-1 \cdot 89$ & $+0 \cdot 81$ & -0.01 & $-1 \cdot 36$ & $+1 \cdot 35$ \\
\hline 7 & $-0 \cdot 18$ & -1.53 & $+1 \cdot 17$ & $+0 \cdot 17$ & $-1 \cdot 18$ & $+1 \cdot 52$ \\
\hline
\end{tabular}

simplest reaction scheme for glucoamylase $(E)$ binding and degrading oligosaccharide substrate $(S)$ in the presence of an inhibitor $(I)$, that can bind to subsites 1 and 2 , can therefore be written

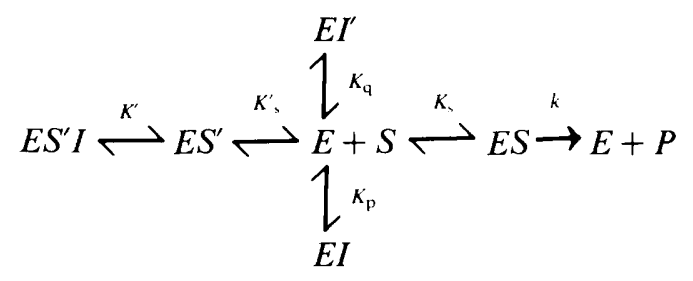

where $E S$ and $E S^{\prime}$ are productive and nonproductive enzyme-substrate complexes respectively, $K_{\mathrm{p}}+K_{\mathrm{p}}^{\prime}$ describe inhibitor binding at subsite 1 , and $K_{\mathrm{q}}$ describes inhibitor binding at subsite 2 . From this reaction scheme (5), the rate equation is

$$
\begin{aligned}
e / v= & (1 / k)\left[1+\left(K_{\mathrm{s}} / K_{\mathrm{s}}^{\prime}\right)\left(1+i / K_{\mathrm{p}}^{\prime}\right)\right] \\
& +\left(K_{\mathrm{s}} / k\right)\left[1+\left(i / K_{\mathrm{q}}\right)+\left(i / K_{\mathrm{p}}\right)\right] 1 / s
\end{aligned}
$$

Thus, if gluconolactone would bind only to subsite 2 (to form $E I^{\prime}$ in the scheme), it would inhibit both productive and nonproductive binding, with no effect on $V$. Binding of gluconolactone to subsite 1 in free enzyme would inhibit only the productive binding, but the formation of $E S^{\prime} I$ would stabilize nonproductive binding and so decrease $V$. 

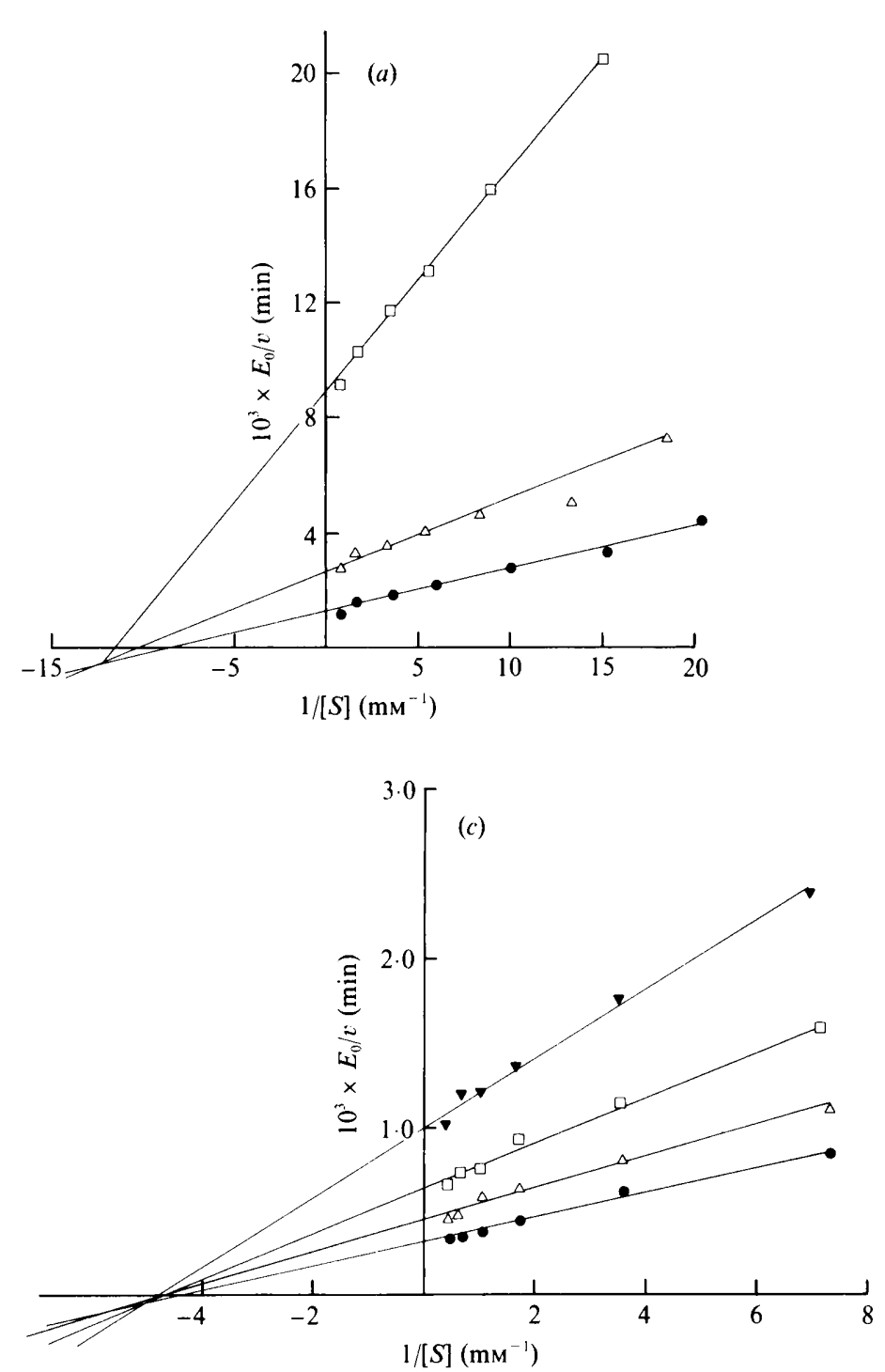
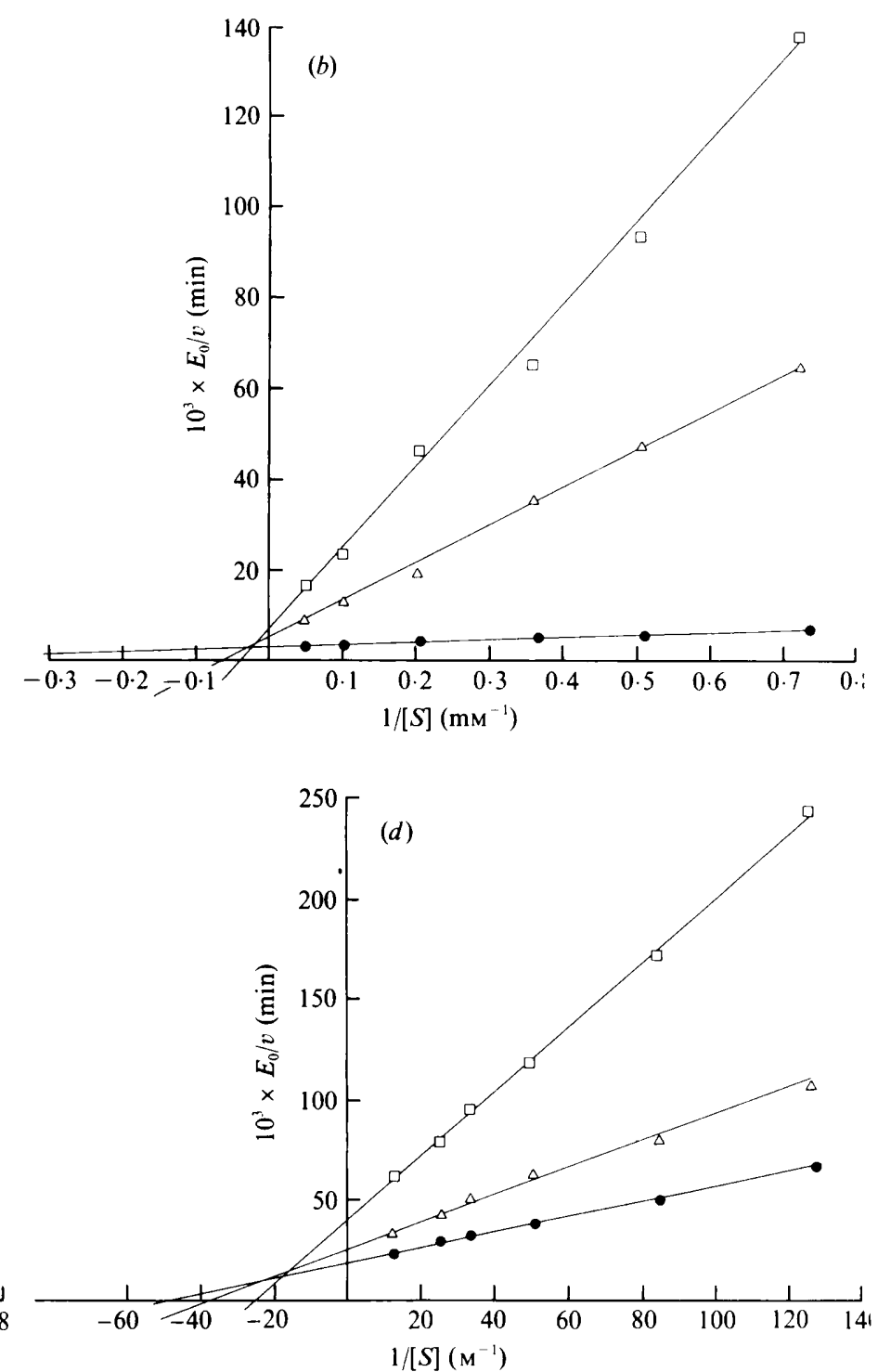

Fig. 1. Lineweaver-Burk double reciprocal plots of the inhibition of glucoamylases by gluconolactone. Inhibition of: $(a)$ glucoamylase $\mathrm{P}(65.2 \mathrm{nM})$ degrading maltohexaose by $0 \mathrm{mM}(\odot), 0.4 \mathrm{mM}(\triangle)$ and $4 \mathrm{mM}(\square)$ gluconolactone; (b) glucoamylase p (81.6 nM) degrading isomaltohexaose by $0 \mathrm{mM}(\circlearrowleft), 4 \mathrm{mM}(\triangle)$ and $10 \mathrm{mM}(\square)$ gluconolactone; (c) glucoamylase $\mathrm{S}(56 \cdot 4 \mathrm{nM})$ degrading maltohexaose by $0 \mathrm{~mm}$ $(\circlearrowleft), 0.4 \mathrm{~mm}(\triangle), 2 \mathrm{~mm}(\square)$ and $4 \mathrm{mM}(\nabla)$ gluconolactone; (d) glucoamylase $\mathrm{S}(1.8 \mu \mathrm{M})$ degrading isomaltohexaose by $0 \mathrm{~mm}(\bullet), 1 \mathrm{~mm}$ $(\triangle)$ and $4 \mathrm{~mm}(\square)$ gluconolactone.

Inhibition by gluconolactone of the degradation of malto- and isomaltohexaose by glucoamylase $\mathrm{P}$ and $\mathrm{S}$ decreases $V$ (Fig. 1), suggesting that both enzymes bind gluconolactone at subsite 1 , like $R$. niveus and $R$. delemar glucoamylases (Hiromi et al., 1983; Ohnishi et al., 1976). For both enzymes the $1 / s$ intercepts are nearly constant for maltohexaose, but change greatly for isomaltohexaose.

By plotting the ordinate intercepts and slopes of the Lineweaver-Burk plots against $i$ (example in Fig. 2), the values of the following set of constants can be obtained for each data set.
From the intercepts

$$
\begin{aligned}
& k_{0}=k K_{\mathrm{s}}^{\prime} /\left(K_{\mathrm{s}}+K_{\mathrm{s}}^{\prime}\right) \\
& K_{\mathrm{i}}^{\prime}=K_{\mathrm{p}}^{\prime}\left(K_{\mathrm{s}}+K_{\mathrm{s}}^{\prime}\right) / K_{\mathrm{s}}
\end{aligned}
$$

From the slopes

$$
K_{\mathrm{i}}=K_{\mathrm{p}} K_{\mathrm{q}} /\left(K_{\mathrm{p}}+K_{\mathrm{q}}\right)
$$

From combination of slopes and intercepts

$$
\begin{aligned}
K_{\mathrm{m}} & =K_{\mathrm{s}} K^{\prime} \mathrm{s} /\left(K_{\mathrm{s}}+K_{\mathrm{s}}^{\prime}\right) \\
K^{\prime \prime}{ }_{\mathrm{i}} & =K^{\prime}{ }_{\mathrm{s}} K_{\mathrm{p}}^{\prime}
\end{aligned}
$$

The values are listed in Table 3. Higher concentrations of inhibitor could not be used, because of the sensitivity 

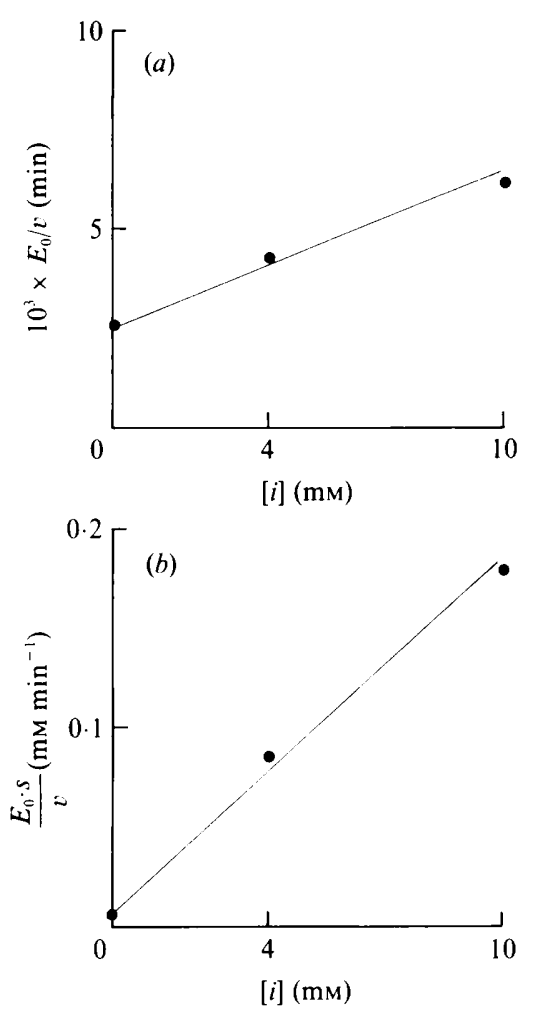

Fig. 2. Example of secondary plots of intercepts $(a)$ and slopes $(b)$ against gluconolactone concentration. The plots are for glucoamylase $P$ with isomaltohexaose.

Table 3. Kinetic constants extracted from the rate equation (6) and estimated from the secondary plots (Fig. 2).

M6, maltohexaose; IM6, isomaltohexaose.

\begin{tabular}{lccccc}
\hline \hline & \multicolumn{2}{c}{ Glucoamylase P } & & \multicolumn{2}{c}{ Glucoamylase S } \\
\cline { 2 - 3 } \cline { 5 - 6 } & M6 & IM6 & & M6 & IM6 \\
\hline$k_{0}\left(\mathrm{~s}^{-1}\right)$ & 11.5 & 6.7 & & 50.5 & 0.84 \\
$K_{\mathrm{m}}(\mathrm{mM})$ & 0.11 & 2.04 & 0.23 & $18 \cdot 0$ \\
$K_{\mathrm{i}}(\mathrm{mM})$ & 1.03 & 0.28 & & 2.41 & 1.20 \\
$K_{i}^{\prime}(\mathrm{mM})$ & 0.78 & 6.25 & & 1.94 & 3.60 \\
$K^{\prime \prime}(\mathrm{mM})$ & 0.086 & 12.8 & & 0.45 & 63.6 \\
\hline \hline
\end{tabular}

limits of the assay and the need to avoid long incubations, which could effect the conversion of gluconolactone (see Methods). Because of the high price of the oligosaccharides, assays using intermediate concentrations of inhibitor were not performed. The slightly different values of $K_{\mathrm{m}}$ and $k_{0}$ compared to those of maltoand isomaltohexaose in Table 1 are due to the use of linear regression analysis in the former and nonlinear regression analysis in the later case. For both enzymes $K^{\prime}$ and $K^{\prime \prime}{ }_{i}$ are much bigger for isomaltohexaose than for maltohexaose. The $K_{\mathrm{i}}$ values are also different for the different substrates. According to scheme (5) they should be the same, since they involve only the dissociation constants of $I$ from free enzyme (namely $K_{\mathrm{p}}$ and $K_{\mathrm{q}}$ ). Apparently this simple reaction scheme does not fully fit the empirical data.

\section{Discussion}

Glucoamylases probably have basically similar reaction mechanisms, because they have very similar amino acid sequences at the active site proposed by Tanaka et al. (1986). Site-directed mutagenesis studies (Sierks et al., 1990) confirm the importance of these sequences.

The relatively high $1,6 / 1,4$-activity ratio of glucoamylase $\mathrm{P}$ results from both its higher $k_{\mathrm{int}}$ towards isomaltooligosaccharides and its lower $k_{\text {int }}$ towards maltooligosaccharides compared with glucoamylase $S$ (Table 1). The high negative free energy of binding of maltooligosaccharides at subsite 1 in glucoamylase $P$ is unique among known glucoamylases (Hiromi, 1970; Tanaka et al., 1983a; Koyama et al., 1984; Monma et al., 1989; Ono et al., 1988; Meagher et al., 1989). For the five glucoamylases purified from Aspergillus niger, this free - energy decreases from about $+2 \cdot 3 \mathrm{~kJ} \mathrm{~mol}^{-1}$ to about $0 \mathrm{~kJ} \mathrm{~mol}^{-1}$ (calculated from the data of Ono et al., 1988) in the same order as increasing relative activity towards dextran 80 (a 1,6-glycosidically linked oligosaccharide). Possibly a large negative value for this free energy favours relatively high activity towards 1,6-linked oligosaccharides.

The gluconolactone inhibition pattern shows that no simple (rigid) enzyme mechanism adequately describes glucoamylases $\mathbf{P}$ and $\mathbf{S}$. This is because the change in the slopes (i.e. the $K_{\mathrm{i}}$ values in Table 3 ) of the LineweaverBurk plots with inhibitor concentration experimentally depends on the nature of the substrate. For any simple scheme, such as scheme (5), the expression for these slopes should include only inhibitor terms involving dissociation constants of the inhibitor from free enzyme. Additional nonproductive enzyme-substrate complexes, involving subsites 3 to $n$ but not subsite 2 , probably occur, but at a much smaller concentration than that utilizing subsite 2 , because $A_{2}$ is about three times bigger than the sum of $A_{3}$ to $A_{7}$ (Table 2). But consideration of such complexes does not alter the expected inhibition pattern: additional terms appear in the intercept of the Lineweaver-Burk plot, but the slope is not changed. If there are two subsites 2 , one for 1,4-and another (possibly overlapping) for 1,6-glucosidically linked substrates, they may bind inhibitor with different dissociation constants, to form two species of $E I^{\prime}$, which, respectively, prevent binding of 1,4- or 1,6-linked substrate. Then there would be two $K_{\mathrm{q}}$ 's in the reaction scheme, and this 
would explain the observed variation of $K_{\mathrm{i}}$ with substrate. Clearly, the same effect could be obtained with a common subsite 2 for 1,4-and 1,6-linked substrates but distinct subsites 1 , which bind $I$ to form two different $E I$ species.

For both glucoamylases $\mathrm{P}$ and $\mathrm{S}, K^{\prime \prime}{ }_{\mathrm{i}}\left[=K_{\mathrm{S}}{ }_{\mathrm{S}}{ }_{\mathrm{p}}\right.$ in scheme (5)] was over 100-fold bigger for isomaltohexaose than maltohexaose, so that either nonproductive substrate binding $\left(K_{\mathrm{s}}^{\prime}\right)$ or binding of gluconolactone to the nonproductive complex $\left(K_{\mathrm{p}}^{\prime}\right)$ is much less favourable for isomaltohexaose than for maltohexaose. Possibly, there is steric interference between gluconolactone bound at subsite 1 and the terminal glucose of isomaltohexaose bound at subsite 2 in the nonproductive mode.

Meagher et al. (1989) and Sierks et al. (1990) have suggested that subsite 2 of glucoamylase from $A$. awamori binds isomaltose in a different way than maltose. This may also be the case with glucoamylases $\mathrm{P}$ and $\mathrm{S}$ from $H$. resinae. The free energy of binding of maltooligosaccharides at subsite 2 of glucoamylases is between -19 and $-22 \mathrm{~kJ} \mathrm{~mol}^{-1}$. The results from $A$. niger (Meagher et al., 1989 ) and the results shown in this paper indicate that the free energy of binding of isomaltooligosacharides to subsite 2 of these enzymes is between -14 and $-15 \mathrm{~kJ} \mathrm{~mol}^{-1}$.

This difference may be due to a difference of one or more hydrogen bonds (corresponding to 2 to $6 \mathrm{~kJ} \mathrm{~mol}^{-1}$ each) and/or a difference in the type of hydrogen bonds (e.g. between two charged groups, 14 to $18 \mathrm{~kJ} \mathrm{~mol}^{-1}$ ) as outlined by Fersht et al. (1985). Thus, it seems justified to assume that the two differently linked oligosaccharides bind differently to the active site of glucoamylase.

Tanaka et al. $(1982,1983 b)$ have suggested that the observed biphasic binding of substrate to the glucoamylase of $R$. niveus represents a fast bimolecular binding of a glucose moiety to subsite 2 followed by a slow, unimolecular 'relocation' to subsite 1. However, an 'induced fit' mechanism seems equally likely, as shown in Fig. 3. Here, the fast step of productive binding is interaction of the penultimate glucose moiety (from the nonreducing end) with subsite 2 to form $E S$. The large negative value of $A_{2}$ makes it reasonable that subsite 2 will have the greatest on-velocity constant. The slow, unimolecular step would represent a conformational change in the enzyme (converting $E S$ to $E S^{*}$ ) by which a tryptophan (presumably Trp-120 in A. awamori; Sierks et al., 1989) and other residues fold around the nonreducing-end glucose to form a stable subsite 1 and change the fluorescence quenching of tryptophan. Hiromi et al. (1982) found that glucose and glucosides, binding to subsite 2, stabilize the gluconolactone-enzyme complex. This can be explained by a conformational change in the enzyme, with gluconolactone binding to a newly formed subsite 1 induced by the binding of glucose or glucosides

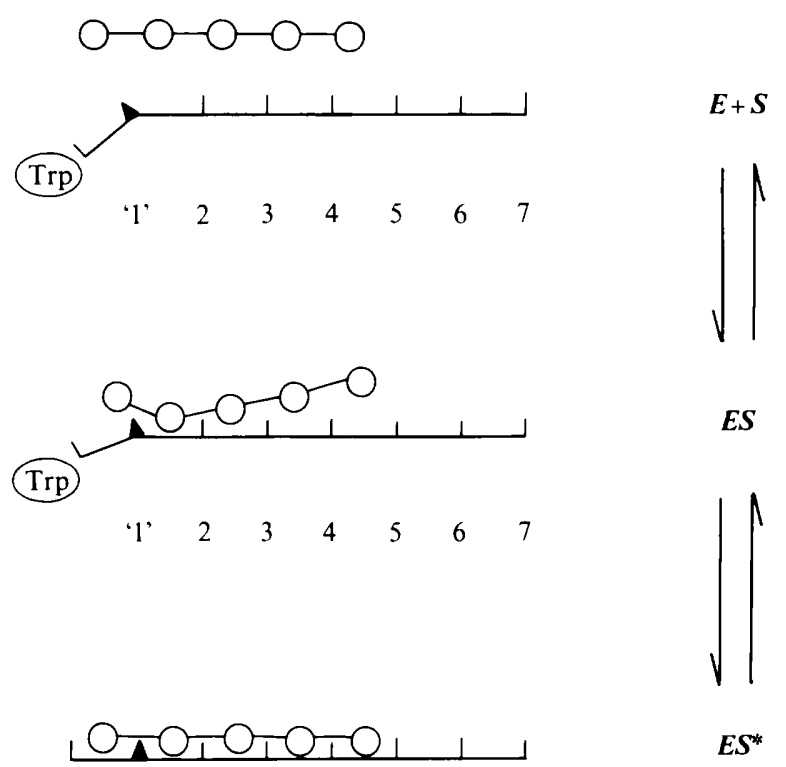

Trp

$$
\begin{array}{lllllll}
1 & 2 & 3 & 4 & 5 & 6 & 7
\end{array}
$$

Fig. 3. Schematic representation of the proposed 'induced fit' model for binding of oligosaccharides to the subsites of glucoamylases. The numbers refer to the subsites starting from the nonreducing-end of the oligosaccharide chain: ' 1 ', unformed subsite $1 ; 1$, newly formed subsite 1 ; $E$, free enzyme; $S$, free substrate; $E S$, loosely bound enzymesubstrate complex; $E S^{*}$, tightly bound productive enzyme-substrate complex; Trp, unquenched fluorescence of tryptophan; Trp, quenched fluorescence of tryptophan.

at subsite 2, to form a ternary gluconolactone-glucose (or glucoside)-enzyme complex. This would also explain the finding (Ono et al., 1964), that the sum of the relative hydrolysis rates of different individual bonds in oligosaccharides is not equal to the overall relative activity. In other words, the environment of the bond affects the hydrolysis rate (Abdullah et al., 1963). This environment could affect the formation of the 'induced fit' structure, as well as its flexibility. A thorough study of the inhibition of the hydrolysis of $p$-nitrophenyl $\alpha$-D-glucoside, phenyl 6-o-acetyl- $\alpha$-maltoside and maltose by different mono- and disaccharides was made by Hiromi et al. $(1973 b)$ with the glucoamylase from $R$. delemar. The type of inhibition was competitive for all analogues examined, indicating that they bind to subsite 2 . None of them resembled gluconolactone. All disaccharides tested had D-glucose as one component, and showed a free energy of binding comparable to that of D-glucose, but much lower than that for maltose. The difference in free energy of binding of these analogues could be explained by the 'induced fit' model, with the formation of subsite 1 being affected by the nature of the non-D-glucose moiety.

It has been proposed that the fluorescence quenching of Rhizopus glucoamylase upon binding of glucono- 
lactone and other substrate analogues may result from a single tryptophan residue that is located near subsite 1 of glucoamylases (Ohnishi et al., 1975; Ohnishi \& Hiromi, 1976; Hiromi et al., 1982). Sierks et al. (1989) found that site-directed mutagenesis of Trp-120 to tyrosine in $A$. awamori glucoamylase decreased the $K_{\mathrm{m}}$ for isomaltose 11 -fold and changed the free energy of binding of maltooligosaccharides at subsite 1 from +2.6 to $-2 \cdot 0 \mathrm{~kJ} \mathrm{~mol}^{-1}$. They propose that Trp-120 is located near subsite 4 , and that it influences binding of $D-$ glucosyl residues at subsites 1 and 2 . Asp-176 has been shown to be located at subsite 1 and to influence the binding energies of maltooligosaccharides at subsite 4 and 5 (Sierks et al., 1990). These results suggest that Trp120 and Asp-176 are responsible for a stabilization of the transition state.

In conclusion, although the physical interpretation of the subsite maps of glucoamylases $P$ and $S$ (and those of other enzymes) depends upon the assumptions made (see Introduction), it seems to be characteristic of enzymes with a high 1,6/1,4- activity ratio that they have a large, negative value for $\boldsymbol{A}_{1}$ with maltooligosaccharides. Gluconolactone binds both enzymes as an inhibitor at subsite 1, but binding to other subsites cannot be excluded. Further, there seem to be distinct gluconolactone binding sites that interfere, respectively, with maltohexaose and isomaltohexaose; i.e. there seem to be distinct subsites 1 (and/or subsites 2) for 1,4- and 1,6linked substrates. Finally, the simple subsite concept for glucoamylases is probably complicated by a stabilization of subsite 1 by occupancy of subsite 2 .

I thank Dr John Londesborough for critical reading of the manuscript, as well as many useful discussions. I also thank Ms Jaana Pekurinen and Ms Tuula Roikonen for technical assistance.

\section{References}

Abdullah, M., Fleming, I. D., Taylor, P. M. \& Whelan, W. J. (1963). Substrate specifity of the amyloglucosidase of Aspergillus niger. Biochemical Journal 89, 35P-36P.

ashikari, T., Nakamura, N., Tanaka, Y., Kiuchi, N., Shibano, Y., Tanaka, T., Awachi, T. \& Yoshizumi, H. (1986). Rhizopus rawstarch-degrading glucoamylase: its cloning and expression in yeast. Agricultural and Biological Chemistry 50, 957-964

Clarke, A. J. \& Svensson, B. (1984). Identification of essential tryptophanyl residue in the primary structure of glucoamylase G2 from Aspergillus niger. Carlsberg Research Communications 49, 559 566.

Fagerström, R., Vainio, A., Suoranta, K., Pakula, T., Kalkkinen, N. \& TORKKeli, H. (1990). Comparison of two glucoamylases from Hormoconis resinae. Journal of General Microbiology 136, 913-920.

Fersht, A. R., Shi, J.-P., Knill-Jones, J., Lowe, D. M., Wilkinson, A. J., Blow, D. M., Brick, P., Carter, P., Waye, M. M. Y. \& Winter, G. (1985). Hydrogen bonding and biological specificity analysed by protein engineering. Nature, London 314, 235-238.
Hiromi, K. (1970). Interpretation of dependency of rate parameters on the degree of polymerization of substrate in enzyme-catalyzed reactions. Evaluation of subsite affinities of exo-enzyme. Biochemical and Biophysical Research Communications 40, 1-6.

Hiromi, K., Hamauzu, Z-I., Takahashi, K. \& ONo, S. (1966a). Kinetic studies on gluc-amylase. II. Competition between two types of substrate having $\alpha-1,4$ and $\alpha-1,6$ glucosidic linkage. Journal of Biochemistry 59, 411-418.

Hiromi, K., Takahashi, K., Hamauzu, Z-I. \& Ono, S. (1966b). Kinetic studies on gluc-amylase. III. The influence of $\mathrm{pH}$ on the rates of hydrolysis of maltose and panose. Journal of Biochemistry 59, 469475 .

Hiromi, K., KawaI, M. \& Ono, S. (1966c). Kinetic studies on glucamylase. IV. Hydrolysis of isomaltose. Journal of Biochemistry 59, $476-480$.

Hiromi, K., Nitta, Y., Numata, C. \& Ono, S. (1973a). Subsite affinities of glucoamylase: Examination of the validity of the subsite theory. Biochimica et Biophysica Acta 302, 362-375.

Hiromi, K., Kawai, M., Suetsugu, N., NitTa, Y. Hosotani, T., NaGaO, A., Nakajima, T. \& ONo, S. $(1973 b)$. Kinetic studies on glucoamylase. VI. Inhibition of substrate analogues. Journal of Biochemistry 74, 935-943.

Hiromi, K., TanaKa, A. \& OHNishi, M. (1982). Fluorometric studies on the binding of gluconolactone, glucose, and glucosides to the subsites of glucoamylase. Biochemistry 21, 102-107.

Hiromi, K., OHNishi, M. \& TanaKa, A. (1983). Subsite structure and ligand binding mechanism of glucoamylase. Molecular and Cellular Biochemistry 51, 79-95.

Itoh, T., Ohtsuki, I., Yamashita, I. \& Fukui, S. (1987). Nucleotide sequence of the glucoamylase gene $G L U I$ in yeast Saccharomycopsis fibuligera. Journal of Bacteriology 169, 4171-4176.

Koyama, T., InOKUChI, N., KikUChi, Y., Shimada, H., Iwama, M., TAKAHASHI, T. \& IRIE, M. (1984). Subsite affinity of a glucoamylase from Aspergillus saitoi. Chemical Pharmacology Bulletin 32, 757-761.

MCCleary, B. V. \& Anderson, M. A. (1980). Hydrolysis of $\alpha-D-$ glucans and $\alpha$-D-glucooligosaccharides by Cladosporium resinae glucoamylases. Carbohydrate Research 86, 77-96.

Meacher, M. M., Nikolov, Z. L. \& Reilly, P. J. (1989). Subsite mapping of Aspergillus niger glucoamylases I and II with malto- and isomaltooligosaccharides. Biotechnology and Bioengineering 34, 681 688 .

Monma, M., Yamamoto, Y. \& Kainuma, K. (1989). Subsite structure of Chlara paradoxa glucoamylase and interaction of the glucoamylase with cyclodextrins. Agricultural and Biological Chemistry 53, 15031508 .

Nikolov, Z. L., Meagher, M. M. \& Reilly, P. J. (1989). Kinetics, equilibria, and modeling of the formation of oligosaccharides from D-glucose with Aspergillus niger glucoamylases I and II. Biotechnology and Bioengineering 34, 694-704.

Nunberg, J. H., Meade, J. H., Cole, G., Lawyer, F. C., McCabe, P., Schweichart, V., Tal, R., Wimtman, V. P., Flatgaard, F. E. \& INNIS, M. A. (1984). Molecular cloning and characterization of the glucoamylase gene of Aspergillus awamori. Molecular and Cellular Biology 4, 2306-2315.

OHNishi, M. \& Hiromi, K. (1976). Studies on the subsite structure of amylases. IV. Tryptophan residues of glucoamylase from Rhizopus niveus studied by chemical modification with N-bromosuccinimid. Journal of Biochemistry 79, 11-16.

OhNishi, M., KeGaI, H. \& Hiromo, K. (1975). Studies on the subsite structure of amylases. I. Interaction of glucoamylases with substrate and analogues studied by difference-spectrophotometry. Journal of Biochemistry 77, 695-703.

Ohnishi, M., Yamashita, T. \& Hiromi, K. (1976). Studies on the subsite structure of amylases. III. Inhibition by gluconolactone of the hydrolysis of maltodextrin catalyzed by glucoamylase from Rhizonus niveus. Journal of Biochemistry 79, 1007-1012.

Ono, S., Hiromi, K. \& Zimbo, M. (1964). Kinetic studies of glucamylase. I. The influence of chain length of linear substrates on the rate parameters. Journal of Biochemistry 55, 315-320.

Ono, K., Shintani, K., Shigeta, S. \& OKa, S. (1988). Comparative studies of various molecular species in Aspergillus niger glucoamylase. Agricultural and Biological Chemistry 52, 1699-1706. 
SCOPES, R. K. (1974). Measurement of protein by spectrophotometry at $205 \mathrm{~nm}$. Analytical Biochemistry 59, 277-282.

ShImIHARA, K. \& TAKAHASHI, T. (1970). An infrared spectrophotometric study on the interconversion and hydrolysis of D-glucono-?and $-\delta$-lactone in deuterium oxide. Biochimica et Biophysica Acta 201, $401-415$.

Sierks, M. R., Ford, C., Reilly, P. \& Svensson, B. (1989). Sitedirected mutagenesis at the active site Trpl 20 of Asnergillus awamori glucoamylase. Protein Engineering 2, 621-625.

Sierks, M. R., Ford, C., Reill.y, P. \& Svensson, B. (1990). Catalytic mechanism of fungal glucoamylases as defined by mutagenesis of Asp176, Glu 179 and Glul 80 in the enzyme from Aspergillus awamori. Protein Engineering 3, 193-198.

Suetsugu, N., Hirooka, E., Yasui, H., Hiromi, K. \& Ono, S. (1973). Kinetic studies on glucoamylase. V. Hydrolyses of phenyl $\alpha$ glucosides and phenyl $\alpha$-maltosides. Journal of Biochemistry 73, 1223 1232.

Svensson, B., Larsen, K., Svedsen, I. \& Boel, E. (1983). The complete amino acid sequence of the glycoprotein, glucoamylase G1, from Aspergillus niger. Carlsberg Research Communications 48, 529-544.

Tanaka, Y., OHNishi, M. \& Hiromi, K. (1982). Stopped-flow kinetic studies on the binding of gluconolactone and maltose to glucoamylase. Biochemistry 21, 107-113.
Tanaka, A., Fukuchi, Y., Ohnishi, M., Hiromi, K., Aibara, S. \& MORITA, Y. (1983a). Fractionation of isozymes and determination of the subsite structure of glucoamylase from Rhizopus niveus. Agricultural and Biological Chemistry 47, 573-580.

Tanaka, A., Yamashita, T., OHNishi, M. \& Hiromi, K. (1983b). Steady-state and transient kinetic studies on the binding of maltooligosaccharides to glucoamylase. Journal of Biochemistry $\mathbf{9 3}$, $1037-1043$.

Tanaka, Y., Ashikari, T., Nakamura, N., KiUchi, N., Shibano, Y., Amachi, T. \& Yoshizumi, H. (1986). Comparison of amino acid sequences of three glucoamylases and their structure-function relationships. Agricultural and Biological Chemistry 50, 965-969.

Wilkinson, G. N. (1961). Statistical estimations in enzyme kinetics. Biochemical Journal 80, 324-332.

Yamashita, I., SuZUKI, K. \& FukUi, S. (1985). Nucleotide sequence of the extracellular glucoamylase gene $S T A 1$ in the yeast Saccharomyces diastaticus. Journal of Bacteriology 161, 567-573.

Yamashita, I., Nakamura, M. \& Fukui, S. (1987). Gene fusion is a possible mechanism underlying the evolution of STA1. Journal of Bacteriology 169, 2142-2149. 